\title{
Defining Adequate Management in Elderly Patients with Candidaemia
}

\author{
Abhijit Mahendra Bal \\ Department of Microbiology, University Hospital Crosshouse, Kilmarnock, UK
}

Dear Editor,

I read with interest the article by Zatta et al. [1] emphasizing the deficiencies in the management of candidaemia in the very elderly group of patients. In our own recently published work, we found that very elderly patients had significantly fewer referrals for echocardiogram and ophthalmoscopy, which are the 2 important performance measures in the management of candidaemia. There were also deficiencies in relation to the timely removal of central venous catheter (CVC) in this group of patients but antifungal therapy was adequate across all age-groups in our institution [2].

Zatta et al. [1] define adequate antifungal treatment in terms of drug susceptibility, dosage, and timely commencement of therapy. However, the authors do not comment on the duration of antifungal therapy. Given that the dataset is from 2011, the IDSA guidelines from 2009 would have been applicable. As per this guidance, antifungal therapy should be offered for 2 weeks from documented clearance of candidaemia [3]. If clearance is not documented, 14 days of therapy from the last positive blood culture can be considered adequate [4]. Patients who die within the period of 14 days while still receiving antifungal therapy should also be considered to have re- ceived adequate therapy. Analysis of data after taking into account the duration of therapy might reveal the true picture pertaining to adequacy of management.

Removal of CVC is generally recommended in all patients with candidaemia [5]. Most patients with CVC will have their central line removed at some point but in the context of candidaemia, timely removal is advisable. The British standard of care recommends removal within $48 \mathrm{~h}$ [6] while the recent EQUAL scoring criteria awards 2 points for removal of CVC within $72 \mathrm{~h}$ and 3 points for removal within $24 \mathrm{~h}$ [7]. It would be interesting to know the timeline of removal of CVC in the patients included in their dataset. While the authors mention the median time to removal in both age-groups, comparing the management of candidaemia in the 2 age-groups in relation to timely removal (at least within $72 \mathrm{~h}$ ) of CVC would provide a better understanding in relation to the deficiencies, if any, in the management of candida bloodstream infection in the very old population.

\section{Conflict of Interest Statement}

The author has no conflicts of interest to declare. 


\section{Funding Source}

The authors did not receive any funding.

\section{Author Contribution}

Designing of the work, drafting the work for important intellectual content, approval of the manuscript, and accountable for the content.

\section{References}

1 Zatta M, Di Bella S, Giacobbe DR, Del Puente F, Merelli M, Azzini AM, et al. Clinical features and mortality of nosocomial candidemia in very old patients: a multicentre Italian study. Gerontology. 2020 Oct 16;66(6):532-41.

$2 \mathrm{Bal}$ AM, Palchaudhuri M. Candidaemia in the elderly: epidemiology, management and adherence to the European confederation of medical mycology quality indicators. Mycoses. 2020 Sep;63(9):892-9.

3 Pappas PG, Kauffman CA, Andes D, Benjamin DK Jr, Calandra TF, Edwards JE Jr, et al. Clinical practice guidelines for the manage- ment of candidiasis: 2009 update by the infectious diseases society of America. Clin Infect Dis. 2009 Mar;48(5):503-35.

4 Ruhnke M, Rickerts V, Cornely OA, Buchheidt D, Glöckner A, Heinz W, et al. Diagnosis and therapy of Candida infections: joint recommendations of the German speaking mycological society and the Paul-Ehrlich-society for chemotherapy. Mycoses. 2011 Jul;54(4):279-310.

5 Cornely OA, Bassetti M, Calandra T, Garbino J, Kullberg BJ, Lortholary O, et al. ESCMID* guideline for the diagnosis and management of Candida diseases 2012: non-neutropenic adult patients. Clin Microbiol Infect. 2012 Dec;18(Suppl 7):19-37.

6 Schelenz S, Barnes RA, Kibbler CC, Jones BL, Denning DW. Standards of care for patients with invasive fungal infections within the United Kingdom: a national audit. J Infect. 2009 Feb;58(2):145-53.

7 Mellinghoff SC, Hoenigl M, Koehler P, Kumar A, Lagrou K, Lass-Flörl C, et al. EQUAL Candida score: an ECMM score derived from current guidelines to measure QUAlity of clinical candidaemia management. Mycoses. 2018 May;61(5):326-30. 